\title{
Flow and Microwave Induced Pellizzari Reactions: Synthesis of Heterocyclic Analogues of the Benzoxazepine Antipsychotic Agents Loxapine and JL-13
}

\author{
Nadeesha Ranasinghe, ${ }^{1}$ Enrico Mongeau, ${ }^{1}$ Gengyang Yuan, ${ }^{1}$ \\ Zachary Minden, ${ }^{1}$ Scott Waldron, ${ }^{1}$ Christopher Patrick Jr., ${ }^{1}$ Farid Fouad, ${ }^{2}$ \\ Sabiha Kappera, ${ }^{2}$ and Graham B. Jones ${ }^{1,3}$ \\ ${ }^{1}$ Department of Chemistry and Chemical Biology, Northeastern University, 360 Huntington Avenue, Boston, MA 02115, USA \\ ${ }^{2}$ Department of Chemistry, Kent State University, East Liverpool, OH 43920, USA \\ ${ }^{3}$ Clinical and Translational Science Institute, Tufts University Medical Center, 800 Washington Street, Boston, MA 02111, USA
}

Correspondence should be addressed to Graham B. Jones; graham.jones@tufts.edu

Received 15 May 2017; Revised 28 September 2017; Accepted 9 October 2017; Published 4 December 2017

Academic Editor: Thierry Besson

Copyright (C) 2017 Nadeesha Ranasinghe et al. This is an open access article distributed under the Creative Commons Attribution License, which permits unrestricted use, distribution, and reproduction in any medium, provided the original work is properly cited.

\begin{abstract}
An expeditious route to fused triazolo-pyrido benzoxazepines has been developed using flow and microwave-mediated cyclization chemistry. A range of substituted aryl hydrazides are coupled with a core chloroimine in good to excellent yield via a Pellizzari type process, producing 1,2,4-triazolo-pyrido [2,3-b] [1,5] benzoxazepines with structural similarity to known antipsychotic agents. Modifications allow for strategically functionalized derivatives, and installation of a fluoro group for use in PET imaging is also demonstrated. Given the affinity of the tricyclic core for 5-HT and dopamine receptors, the derivatives are expected to find utility in CNS research.
\end{abstract}

\section{Introduction}

Heterocyclic derivatives of the benzodiazepine and benzoxazepine class are of considerable interest in medicinal chemistry due to their pharmacologic and metabolic profiles $[1,2]$. Prominent examples include the anxiolytic Alprazolam $\left(\mathrm{Xanax}^{\circledR}\right)$, Loxapine, and the atypical antipsychotic JL13, which has been investigated as a next-generation clozapine analogue [3-5]. One of the pharmacologic targets of the agents is the $5-\mathrm{HT}_{2 \mathrm{a}}$ receptor, and a number of heterocyclic analogues demonstrate high affinity including Asenapine which despite its altered geometry compares favorably to traditional agents Loxapine and Clozapine [6] (see Scheme 1). Given promising preclinical data reported for JL13 [7-9], we elected to study the potential of additional heterocyclic variants which might be readily accessible using efficient coupling strategies and which also highlights the benefits of green chemistry approaches $[10,11]$. Given the functional flexibility of the triazole group and its presence in a number of pharmacologically active molecules [12], we sought to investigate use of the Pellizzari method [13], which relies on one pot coupling, and closure of imino chlorides with substituted hydrazides.

\section{Results and Discussion}

For initial studies we designed a series of hybrid triazolebenzoxazepine mimics of Loxapine and JL13, investigating the benefit of microwave and flow-mediated Pellizzari reactions as green approaches to these classes. A chloropyridyl amide substrate 1 was firstly prepared by condensation of 2-chloro nicotinoyl chloride with 2-amino phenol (DIPEA, 94\%). This was subjected to microwave accelerated basemediated intramolecular closure to form 2 (Scheme 2) with $\mathrm{KOtBu}(90 \%)$ preferable to both $\mathrm{NaOH}(72 \%)$ and $\mathrm{NaH}(59 \%)$ with a 5 min irradiation. The cyclic amide was converted to the corresponding chloroimine, allowing us to probe the key 
<smiles>CN1CCN(C2=Nc3cc(Cl)ccc3Nc3ccccc32)CC1</smiles>

Clozapine<smiles>CN1CCN(C2=Nc3ccccc3Oc3ccc(Cl)cc32)CC1</smiles>

Loxapine<smiles>CN1CCN(C2=Nc3cc(Cl)ccc3Oc3ncccc32)CC1</smiles>

JL13<smiles>CN1CC2c3ccccc3Oc3ccc(Cl)cc3C2C1</smiles>

Asenapine<smiles>Cc1nnc2n1-c1ccc(Cl)cc1C(c1ccccc1)=NC2</smiles>

Alprazolam

Scheme 1

TABLE 1: Intramolecular cyclization to form triazolo benzoxepines 3 (\% isolated yield).

\begin{tabular}{|c|c|c|c|c|c|}
\hline Entry & 3 & $\mathrm{Ar}$ & Thermal $^{\mathrm{a}}$ & $\mathrm{MW}^{\mathrm{b}}$ & Flow $^{c}$ \\
\hline 1 & $3 a$ & $\mathrm{Ph}$ & 38 & 54 & 57 \\
\hline 2 & $3 b$ & $2-\mathrm{Cl}-\mathrm{C}_{6} \mathrm{H}_{4}$ & 48 & 86 & 78 \\
\hline 3 & $3 c$ & $4-\mathrm{Cl}-\mathrm{C}_{6} \mathrm{H}_{4}$ & 66 & 96 & 84 \\
\hline 4 & $3 d$ & $2-\mathrm{OH}-\mathrm{C}_{6} \mathrm{H}_{4}$ & 29 & 89 & $>99$ \\
\hline 5 & $3 e$ & $4-\mathrm{OH}-\mathrm{C}_{6} \mathrm{H}_{4}$ & 41 & 67 & 72 \\
\hline 6 & $3 f$ & $3-\mathrm{OMe}-\mathrm{C}_{6} \mathrm{H}_{4}$ & 28 & 92 & 82 \\
\hline 7 & $3 g$ & 3-pyridyl & 57 & 84 & 98 \\
\hline 8 & $3 h$ & $2-\mathrm{F}-\mathrm{C}_{6} \mathrm{H}_{4}$ & 25 & 88 & $>99$ \\
\hline 9 & $3 \mathbf{i}$ & 3-F- $\mathrm{C}_{6} \mathrm{H}_{4}$ & 32 & 83 & 85 \\
\hline 10 & $3 \mathbf{j}$ & $4-\mathrm{Me}-\mathrm{C}_{6} \mathrm{H}_{4}$ & 39 & 98 & 88 \\
\hline 11 & $3 \mathbf{k}$ & $3-\mathrm{NO}_{2}-\mathrm{C}_{6} \mathrm{H}_{4}$ & 17 & 81 & 85 \\
\hline 12 & 31 & $3-\mathrm{Cl}-\mathrm{C}_{6} \mathrm{H}_{4}$ & 44 & 80 & - \\
\hline
\end{tabular}

${ }^{\mathrm{a}} n$ - $\mathrm{BuOH}, \Delta, 118^{\circ} \mathrm{C}, 6 \mathrm{~h} ;{ }^{\mathrm{b}} 300 \mathrm{~W}, 200^{\circ} \mathrm{C}, n-\mathrm{BuOH} 15 \mathrm{~min} .{ }^{\mathrm{c}} \mathrm{Both}$ pumps of the reactor were set to flow at equal rates $(2 \mu \mathrm{l} / \mathrm{min})$ at $190^{\circ} \mathrm{C}$ with a solution of 5 chlorobenzo[b]pyrido[3,2-f] [1,4] oxazepine $(0.04 \mathrm{M})$ and hydrazide $(0.04 \mathrm{M})$ in $n$ - $\mathrm{BuOH}$ with residence time of $2.5 \mathrm{~min}$. Experiments represent $n>3$.

triazole forming reaction to produce 3 (All new compounds were fully characterized by appropriate spectroscopic and analytical techniques.). A range of aryl hydrazide substrates were probed under thermal, microwave, and flow conditions at $100 \mathrm{mg}$ scale and the results are summarized in Table 1.

Though moderate conversion was observed within $6 \mathrm{~h}$ of conventional thermolysis, the MW methods allowed very high yields of product within 15 mins with $n$-butanol being the most effective solvent. Essentially comparable, flowmediated reactions (conducted using a Chemtrix Labtrix S1 system equipped with T-mixer) in some cases produced even higher yields, a presumed consequence of improved mixing interactions [14].

Given literature reports citing the importance of both basic amino functionality and a hydrophobic substituent (e.g., chloroaryl group) for optimal receptor binding $[15,16]$, a more elaborate substrate 5 was also prepared from hydrazide 4 (readily available from $m$-chloro- $o$-toluic acid), leading to the target compound in $87 \%$ yield (see Scheme 3 ). Given the impressive results with the MW Pellizzari reaction, a second family of analogues were prepared which contain the chloroaryl functionality on the parent tricyclic ring system. Amide 6 (prepared from 2-chloro nicotinoyl chloride and 2-amino-4-chloro phenol) was subjected to base induced oxepane formation and subsequent chlorination to give 7 in good yield (Scheme 4). Microwave induced Pellizzari reactions were conducted with various hydrazides to give substituted analogues $\mathbf{8}$.

A range of decorated analogues 9-16 were produced in good to excellent yield (see Scheme 6), which, coupled with those depicted in Table 1, will allow insightful structureactivity studies to be performed with binding assays against key receptors $\left(5-\mathrm{HT}_{2 \mathrm{a}}, 5-\mathrm{HT}_{2 \mathrm{c}}, \mathrm{D}_{1}, \mathrm{D}_{2}\right.$, and $\mathrm{D}_{4.2}$ being of importance, with the $5-\mathrm{HT}_{2 \mathrm{a}} / \mathrm{D}_{2}$ ratio especially significant) [17]. In a preliminary assay, compound 5 showed a $K_{i}$ of $16 \mu \mathrm{M}$ against $5-\mathrm{HT}_{2 \mathrm{a}}$, which bodes well for in depth assays [18]. Finally, and with a potential view to studying biodistribution of these ligands via in vivo imaging methods, proof 

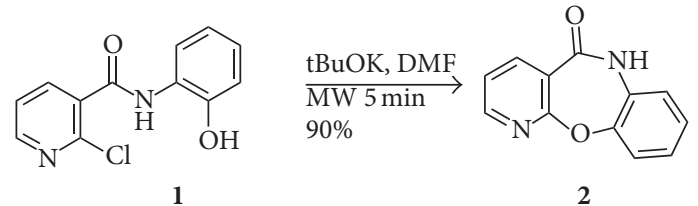
(1) $\mathrm{POCl}_{3}, N, N$-DMA $\mathrm{PhMe} 3 \mathrm{~h}$ $93 \%$
(2) $\mathrm{Ar}-\mathrm{CONHNH}_{2}$ $n-\mathrm{BuOH}, \Delta$

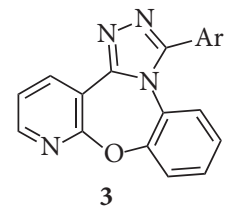

SCHEME 2: MW and flow based synthesis of triazolo-pyrido-benzoxazepines.

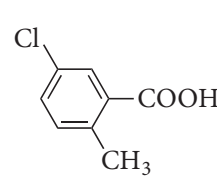

(1) $\mathrm{NaH}, \mathrm{MeI}, \mathrm{DMF} 95 \%$

(2) $\mathrm{CCl}_{4}, \mathrm{AIBN}, \mathrm{NBS} 93 \%$

(3) $\mathrm{Me}_{2} \mathrm{NH}, \mathrm{Et}_{2} \mathrm{O} 94 \%$

(4) $\mathrm{NH}_{2} \mathrm{NH}_{2} \cdot \mathrm{H}_{2} \mathrm{O}$ EtOH $86 \%$

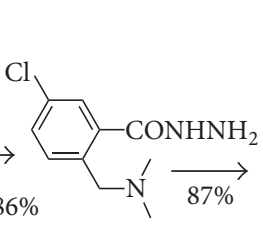

4

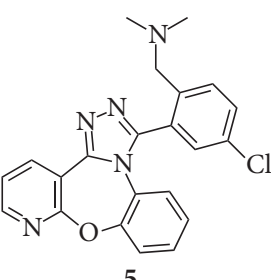

5

SCHEMe 3

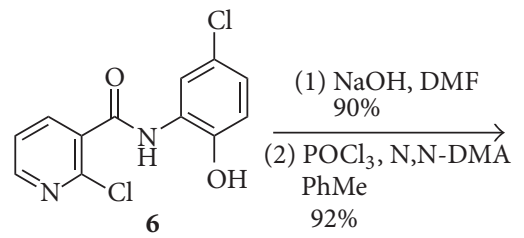<smiles>ClC1=Nc2cc(Cl)ccc2Oc2ncccc21</smiles><smiles>[R]OC(=O)CN</smiles><smiles>[R]c1nnc2n1-c1cc(Cl)ccc1Oc1ncccc1-2</smiles>

SCHeme 4: Modification of core for MW Pellizzari route to chlorinated series.

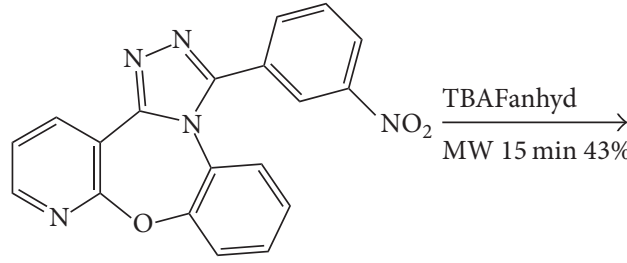

17<smiles>Fc1cccc(-c2nnc3n2-c2ccccc2Oc2ncccc2-3)c1</smiles>

18

Scheme 5: MW induced fluorodenitration.

of principal was demonstrated for installation of a fluoro group via nucleophilic displacement. Following extensive optimization of parameters (temp, time, and stoichiometry), nitroarene $\mathbf{1 7}$ was subjected to MW induced fluorodenitration to give 18 in good yield within 15 mins at $140^{\circ} \mathrm{C}$ using 2 equivalents of TBAF in DMSO (Scheme 5) [19]. Given the half-life of its $\gamma$ emitting sister ${ }^{18} \mathrm{~F}$ isotope ( 120 mins) this rapid transformation is compatible with late stage labeling for PET imaging.

\section{Conclusions}

Both MW and flow based Pellizzari reactions were effective and efficient for the production of new classes of substituted triazolo-pyrido fused benzoxazepines. The route provides access to compounds which may be useful probes of the 5-HT and dopamine receptors, based on similarity to known antipsychotic agents. A specimen compound shows measurable affinity for one of the receptor subtypes and derivatization of the core via nucleophilic fluorination provides a means to utilize the class in PET imaging studies. A comprehensive screening of these agents will be reported in due course.

\section{Appendix}

\section{A. General Experimental Procedures}

All solvents were of reagent or anhydrous grade quality and purchased from Sigma-Aldrich, Alfa Aesar, or Fisher Scientific. All reagents were purchased from Sigma-Aldrich, Alfa Aesar, Fisher Scientific, or Oakwood Chemical, unless otherwise stated. All deuterated solvents were purchased from Cambridge isotopes. Analytical thin-layer chromatography (TLC) was performed on precoated glass-backed plates (EMD TLC Silica gel $60 \mathrm{~F}_{254}$ ) and visualized using a UV lamp $(254 \mathrm{~nm})$ and potassium permanganate stain. Silica gel for manual flash chromatography was high purity grade 


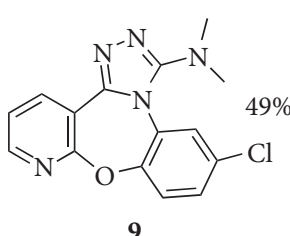

9<smiles>CN1CCN(Cc2nnc3n2-c2cc(Cl)ccc2Oc2ncccc2-3)CC1</smiles>

12<smiles>CN(C)Cc1nnc2n1-c1cc(Cl)ccc1Oc1ncccc1-2</smiles>

10<smiles>Clc1ccc2c(c1)-n1c(-c3cccs3)nnc1-c1cccnc1O2</smiles>

13<smiles>CN1CCN(c2nnc3n2-c2cc(Cl)ccc2Oc2ncccc2-3)CC1</smiles>

11<smiles>Clc1ccc2c(c1)-n1c(-c3ccco3)nnc1-c1cccnc1O2</smiles>

14<smiles>Nc1ccc(-c2nnc3n2-c2cc(Cl)ccc2Oc2ncccc2-3)cc1</smiles>

15<smiles>CN1CCN(Cc2ccc(-c3nnc4n3-c3cc(Cl)ccc3Oc3ncccc3-4)cc2)CC1</smiles>

16

SCHEME 6

40-63 $\mu \mathrm{m}$ pore size and purchased from Sigma-Aldrich. Yields refer to purified and spectroscopically pure compounds. Unless otherwise noted, compounds were recrystallized from toluene-hexanes. ${ }^{1} \mathrm{H}$ NMR (400 MHz, DMSO-d6) and ${ }^{13} \mathrm{C}$ NMR (100 MHz, DMSO-d6) spectra were obtained with a Varian instrument.

A.1. Synthesis of 5-Chlorobenzo[b]pyrido[3,2-f][1,4]oxazepine. A mixture of amide, benzo[b]pyrido[3,2- $f][1,4]$ oxazepine$5(6 \mathrm{H})$-one $(2,1.08 \mathrm{~g}, 5.08 \mathrm{mmol})$, phosphorous oxychloride $(1.42 \mathrm{~mL}, 15.26 \mathrm{mmol})$, and N,N-dimethylaniline $(2.57 \mathrm{~mL}$, $20.32 \mathrm{mmol})$ in dry toluene $(10 \mathrm{~mL})$ was heated at reflux for $2 \mathrm{~h}$. The reaction mixture was cooled to ambient temperature and excess solvents were evaporated under reduced pressure. The resulting residue was dissolved in THF $(20 \mathrm{ml})$ and $\mathrm{Na}_{2} \mathrm{CO}_{3}$ (30 mL of $2 \mathrm{M}$ solution) and heated at $80^{\circ} \mathrm{C}$ for $1 \mathrm{~h}$. The reaction mixture was cooled to ambient temperature and THF was removed under reduced pressure. The resulting aqueous layer was extracted with EtOAc $(3 \times 120 \mathrm{ml})$. The combined organic layers were washed with brine $(1 \times 100 \mathrm{ml})$ and dried over $\mathrm{MgSO}_{4}$ and solvents were evaporated. The resulting residue was purified by column chromatography using 5\% $\mathrm{MeOH}$ in dichloromethane to afford the title compound $(1.09 \mathrm{~g}, 93 \%)$ as a light green solid m.p. $141.9-142.7^{\circ} \mathrm{C}$; ${ }^{1} \mathrm{H}$ NMR: $\delta 8.49(\mathrm{dd}, J=5.2,8 \mathrm{~Hz}, 1 \mathrm{H}), 8.26(\mathrm{dd}, J=2,7.2 \mathrm{~Hz}$, $1 \mathrm{H}), 7.45(\mathrm{dd}, J=5.2,8 \mathrm{~Hz}, 1 \mathrm{H}), 7.33(\mathrm{~d}, 8 \mathrm{~Hz}, 1 \mathrm{H}), 7.24-7.14$ $(\mathrm{m}, 3 \mathrm{H})$ ppm; ${ }^{13} \mathrm{C}$ NMR: $\delta 164.4,162.3,152.4,148.1,142.4$, 130.5, 126.3, 125.3, 122.5, 121.8, 121.7, 120.0 ppm. HRMS calc. for $\mathrm{C}_{12} \mathrm{H}_{7} \mathrm{ClN}_{2} \mathrm{O}: 230.0246$ obs: 230.0244 .

A.1.1. General Procedure for Synthesis of 3 in MW. 5-Chlorobenzo[b]pyrido $[3,2-f][1,4]$ oxazepine $(0.14 \mathrm{~g}, 0.61 \mathrm{mmol}, 1 \mathrm{eq})$ and hydrazide $(0.61 \mathrm{mmol}, 1 \mathrm{eq})$ were dissolved in 1-butanol
$(3 \mathrm{~mL})$ in a $10 \mathrm{~mL}$ glass microwave tube. The vial was capped with a CEM corp. PL cap (SP1318A) and stirred in the cavity of a CEM Discover ${ }^{\circledR}$ Lab Mate reactor set at $200^{\circ} \mathrm{C}$ for $15 \mathrm{~min}(300 \mathrm{~W}, 250 \mathrm{psi})$. The contents of the tube were poured onto crushed ice/cold water $(30 \mathrm{~mL})$ and extracted with EtOAc $(3 \times 25 \mathrm{~mL})$. The combined organic layers were washed with brine $(1 \times 25 \mathrm{~mL})$ and dried over $\mathrm{MgSO}_{4}$. Removal of solvents under reduced pressure followed by silica gel chromatography $\left(\mathrm{CH}_{2} \mathrm{Cl}_{2}\right.$, with $1-10 \% \mathrm{MeOH}$ gradient) afforded the corresponding adduct 3 .

A.1.2. General Procedure for Synthesis of 3 in Flow [20, 21]. Synthesis was performed using a ChemTrix Labtrix S1 flow chemistry reactor containing a T-mixer [14]. Syringe A was loaded with a $0.04 \mathrm{M}$ solution of 5-chlorobenzo[b]pyrido[3,2-f][1,4] oxazepine in $t$-butanol and syringe B was loaded with a $0.04 \mathrm{M}$ solution of corresponding hydrazide in $t$-butanol. Syringes A and B were pumped through a $10 \mu \mathrm{L}$ glass microreactor heated to $190^{\circ} \mathrm{C}$ with a combined flow rate of $4 \mu \mathrm{L} / \mathrm{min}$. The output collected was poured over crushed ice/cold water $(20 \mathrm{~mL})$ and extracted with EtOAc $(3 \times 20 \mathrm{~mL})$. The combined organic layers were washed with brine $(1 \times 20 \mathrm{~mL})$ and dried over $\mathrm{MgSO}_{4}$. The solvents were removed under reduced pressure and the crude product was purified by silica gel chromatography $\left(\mathrm{CH}_{2} \mathrm{Cl}_{2}\right.$, with $1-10 \% \mathrm{MeOH}$ gradient).

A.2.5-Chlorobenzo[b]pyrido[3,2-f][1,4]oxazepine. To a cooled solution of $2(1.08 \mathrm{~g}, 5.08 \mathrm{mmol})$ in dry toluene $(10 \mathrm{~mL})$ $\mathrm{POCl}_{3}$ was added $(1.42 \mathrm{~mL}, 15.26 \mathrm{mmol})$ in a $50 \mathrm{~mL}$ round bottom flask under an atmosphere of argon followed by N,Ndimethylaniline $(2.57 \mathrm{~mL}, 20.32 \mathrm{mmol})$. After refluxing for $2 \mathrm{~h}$ the mixture was concentrated under reduced pressure to 
remove excess $\mathrm{POCl}_{3}$ and $\mathrm{N}, \mathrm{N}$-dimethylaniline. The resulting mixture was dissolved in THF $(20 \mathrm{~mL})$ and $\mathrm{Na}_{2} \mathrm{CO}_{3}(30 \mathrm{~mL}$ of a $2 \mathrm{M}$ solution) and heated at $80^{\circ} \mathrm{C}$. After $1 \mathrm{~h}$ the mixture was cooled to room temperature and THF was removed under reduced pressure. The resulting aqueous layer was extracted to $\mathrm{EtOAc}(3 \times 120 \mathrm{~mL})$ and the combined organic layers were washed with brine $(1 \times 100 \mathrm{~mL})$, dried over $\mathrm{MgSO}_{4}$, and concentrated in vacuo. The residue was purified using silica gel chromatography $\left(5 \% \mathrm{MeOH}\right.$ in $\mathrm{CH}_{2} \mathrm{Cl}_{2}$ ) to afford 5-chlorobenzo[ $b]$ pyrido $[3,2-f][1,4]$ oxazepine as a light green solid (1.09 g, 93\%). mp: $141.9-142.7^{\circ} \mathrm{C} ;{ }^{1} \mathrm{H}$ NMR: ppm 8.49 $(\mathrm{dd}, J=8,5.2 \mathrm{~Hz}, 1 \mathrm{H}), 8.26(\mathrm{~d}, J=7.2 \mathrm{~Hz}, 1 \mathrm{H}), 7.45(\mathrm{dd}, J=$ $7.8,4.8 \mathrm{~Hz}, 1 \mathrm{H}), 7.33(\mathrm{~d}, J=7.2 \mathrm{~Hz}, 1 \mathrm{H}), 7.24-7.14(\mathrm{~m}, 3 \mathrm{H}){ }^{13} \mathrm{C}$ NMR: ppm 164.4, 162.2, 152.3, 148.0, 142.3, 130.5, 130.3, 126.3, $125.5,122.4,121.8,119.9 \mathrm{MS}(\mathrm{ESI}+), m / z \mathrm{C}_{12} \mathrm{H}_{8} \mathrm{ClN}_{2} \mathrm{O}(\mathrm{M}+$ $\mathrm{H})^{+}$calcd: 231.03 obsd: 232.0 .

A.2.1. Synthesis of $\mathbf{5}$ from Hydrazide 4. Compound $\mathbf{4}$ $(0.615 \mathrm{mmol}, 0.14 \mathrm{~g})$ and 5 -chlorobenzo[b]pyrido[3,2-f][1, 4] oxazepine $(0.615 \mathrm{mmol}, 0.14 \mathrm{~g})$ were dissolved in $3 \mathrm{~mL}$ of $\mathrm{n}$ $\mathrm{BuOH}$ in a $10 \mathrm{~mL}$ glass microwave vial and subjected to $\mathrm{MW}$ irradiation at $200^{\circ} \mathrm{C}$ for $15 \mathrm{~min}$. The resulting mixture was concentrated in vacuo and purified by silica gel chromatography $\left(1 \% \mathrm{MeOH}\right.$ in $\left.\mathrm{CH}_{2} \mathrm{Cl}_{2}\right)$ to afford 5 as a pale yellow solid (0.21 g, 87\%). m.p. $140-143^{\circ} \mathrm{C}^{1} \mathrm{H}$ NMR: ppm 8.58 (dd, $J=7.7$, $1.8 \mathrm{~Hz}, 1 \mathrm{H}), 8.48(\mathrm{dd}, J=4.8,1.8 \mathrm{~Hz}, 1 \mathrm{H}), 7.61(\mathrm{~d}, J=8 \mathrm{~Hz}$, $1 \mathrm{H}), 7.45-7.39(\mathrm{~m}, 4 \mathrm{H}), 7.33-7.31(\mathrm{~m}, 1 \mathrm{H}), 7.01(\mathrm{t}, J=7.7 \mathrm{~Hz}$, $1 \mathrm{H}), 6.82(\mathrm{~d}, J=8 \mathrm{~Hz}, 1 \mathrm{H}), 3.89(\mathrm{~s}, 2 \mathrm{H}), 2.25(\mathrm{~s}, 6 \mathrm{H}) ;{ }^{13} \mathrm{C}$ NMR: ppm 158.9, 158.3, 151.0, 133.6, 133.5, 132.0, 131.9, 131.6, $131.5,131.2,130.8,130.3,130.2,130.1,126.5,124.6,124.0,122.9$, 60.7, 45.4; HRMS (ESI+), $m / z \mathrm{C}_{22} \mathrm{H}_{19} \mathrm{ClN}_{5} \mathrm{O}(\mathrm{M}+\mathrm{H})^{+}$calc: 404.1278 obs: 404.1282 .

A.3. Spectroscopic Data [See Supplemental Information for

${ }^{13} \mathrm{C}$ Assignments]. See Supplemental Information for ${ }^{13} \mathrm{C}$ assignments in Supplementary Material available online at https://doi.org/10.1155/2017/8147421.

3a (R=Ph): m.p. $246-247^{\circ} \mathrm{C} ;{ }^{1} \mathrm{H}$ NMR: ppm 8.59 (d, $J=$ $7.2 \mathrm{~Hz}, 1 \mathrm{H}), 8.48-8.47(\mathrm{~m}, 1 \mathrm{H}), 7.96-7.95(\mathrm{~m}, 1 \mathrm{H}), 7.64(\mathrm{~d}, J=$ $7.6 \mathrm{~Hz}, 2 \mathrm{H}), 7.53-7.45(\mathrm{~m}, 3 \mathrm{H}), 7.40-7.38(\mathrm{~m}, 2 \mathrm{H}), 7.05(\mathrm{t}, J=$ $7.7 \mathrm{~Hz}, 1 \mathrm{H}), 6.87$ (d, $J=8 \mathrm{~Hz}, 1 \mathrm{H}) ;{ }^{13} \mathrm{C}$ NMR: ppm 161.6, 153.9, 151.0, 133.0, 131.0, 130.8, 130.3, 129.3, 129.2, 126.8, 126.4, 125.9, $124.4,124.2$, 122.9, 122.0, 118.6, 115.2, 114.9; HRMS (ESI+), $m / z$ $\mathrm{C}_{19} \mathrm{H}_{13} \mathrm{~N}_{4} \mathrm{O}(\mathrm{M}+\mathrm{H})^{+}$calc: 313.1089 obs: 313. 1092 .

3b $\left(\mathrm{R}=0-\mathrm{ClC}_{6} \mathrm{H}_{5}\right)$ : m.p. $241-244^{\circ} \mathrm{C} ;{ }^{1} \mathrm{H}$ NMR: ppm 8.57 $(\mathrm{d}, J=7.2 \mathrm{~Hz}, 1 \mathrm{H}), 8.48(\mathrm{~d}, J=4.4 \mathrm{~Hz}, 1 \mathrm{H}), 7.77(\mathrm{~d}, J=4.4 \mathrm{~Hz}$, $1 \mathrm{H}), 7.62(\mathrm{~d}, J=8.1 \mathrm{~Hz}, 1 \mathrm{H}), 7.52-7.34(\mathrm{~m}, 5 \mathrm{H}), 7.00(\mathrm{t}, J=$ $8 \mathrm{~Hz}, 1 \mathrm{H}), 6.74(\mathrm{~d}, J=8 \mathrm{~Hz}, 1 \mathrm{H}) ;{ }^{13} \mathrm{C}$ NMR: ppm 162.0, 161.8, $151.1,150.5,150.3,139.6,134.2,132.7,132.3,130.6,130.3,127.8$, $127.6,126.8,126.5,124.2,123.5,122.8,115.3$; HRMS (ESI+), $m / z$ $\mathrm{C}_{19} \mathrm{H}_{12} \mathrm{ClN}_{4} \mathrm{O}(\mathrm{M}+\mathrm{H})^{+}$calc: 347.0700 obs: 347.0694 .

3c $\left(\mathrm{R}=p-\mathrm{ClC}_{6} \mathrm{H}_{5}\right)$ : m.p. $236-238^{\circ} \mathrm{C} ;{ }^{1} \mathrm{H}$ NMR: ppm 8.60 $(\mathrm{d}, J=7.2 \mathrm{~Hz}, 1 \mathrm{H}), 8.50(\mathrm{~d}, J=4.4 \mathrm{~Hz}, 1 \mathrm{H}), 7.67(\mathrm{~d}, J=$ $8.1 \mathrm{~Hz}, 1 \mathrm{H}), 7.60$ (d, $J=8.8 \mathrm{~Hz}, 2 \mathrm{H}), 7.46-7.40$ (m, 4H), 7.10 $(\mathrm{t}, J=7.7 \mathrm{~Hz}, 1 \mathrm{H}), 6.88(\mathrm{~d}, J=8 \mathrm{~Hz}, 1 \mathrm{H}) ;{ }^{13} \mathrm{C}$ NMR: ppm $152.7,151.1,147.5,146.2,141.4,139.8,137.1,136.4,132.8,130.5$, 129.6, 126.5, 125.3, 124.3, 123.1, 116.3, 115.1; HRMS (ESI+), $m / z$ $\mathrm{C}_{19} \mathrm{H}_{12} \mathrm{~N}_{4} \mathrm{ClO}(\mathrm{M}+\mathrm{H})^{+}$calc: 347.0700 obs: 347.0706 .
$3 \mathbf{e}\left(\mathrm{R}=p \mathrm{OHC}_{6} \mathrm{H}_{5}\right)$ : m.p. $264-265^{\circ} \mathrm{C} ;{ }^{1} \mathrm{H}$ NMR: ppm 8.61 $(\mathrm{d}, J=7.3 \mathrm{~Hz}, 1 \mathrm{H}), 8.50(\mathrm{~d}, J=4.4 \mathrm{~Hz}, 1 \mathrm{H}), 8.10(\mathrm{~d}, J=8 \mathrm{~Hz}$, $1 \mathrm{H}), 7.51(\mathrm{~d}, J=8 \mathrm{~Hz}, 2 \mathrm{H}), 7.42(\mathrm{~d}, J=8.1 \mathrm{~Hz}, 2 \mathrm{H}), 7.10(\mathrm{t}, J=$ $7.2 \mathrm{~Hz}, 1 \mathrm{H}), 6.95-6.93$ (m, 3H), 6.69 (bs, $1 \mathrm{H}) ;{ }^{13} \mathrm{C}$ NMR: ppm 202.6, 161.7, 161.3, 152.0, 151.6, 150.1, 141.0, 131.3, 131.2, 131.1, $130.85,128.5,126.5,124.0,123.9,121.4,116.5$; HRMS (ESI+), $m / z \mathrm{C}_{19} \mathrm{H}_{13} \mathrm{~N}_{4} \mathrm{O}_{2}(\mathrm{M}+\mathrm{H})^{+}$calc: 329.1039 obs: 329.1038 .

3f $\left(\mathrm{R}=m \mathrm{OCH}_{3} \mathrm{C}_{6} \mathrm{H}_{5}\right)$ : m.p. $255-256{ }^{\circ} \mathrm{C} ;{ }^{1} \mathrm{H}$ NMR: ppm $8.54(\mathrm{~d}, J=8 \mathrm{~Hz}, 1 \mathrm{H}), 8.43(\mathrm{~d}, J=7.2 \mathrm{~Hz}, 1 \mathrm{H}), 7.61-7.59(\mathrm{~m}$, $1 \mathrm{H}), 7.37-7.32(\mathrm{~m}, 3 \mathrm{H}), 7.19(\mathrm{~s}, 1 \mathrm{H}), 7.10-7.08(\mathrm{~m}, 1 \mathrm{H}), 7.05-6.98$ $(\mathrm{m}, 2 \mathrm{H}), 6.92(\mathrm{~d}, J=8 \mathrm{~Hz}, 1 \mathrm{H}), 3.75(\mathrm{~s}, 3 \mathrm{H}) ;{ }^{13} \mathrm{C}$ NMR: ppm 161.6, 160.0, 154.3, 150.8, 150.4, 130.3, 130.2, 126.3, 125.9, 127.8, $127.5,126.3,125.9,124.0,122.9,121.6,117.0,115.1,114.2,55.6$; HRMS (ESI+), $m / z \mathrm{C}_{20} \mathrm{H}_{15} \mathrm{~N}_{4} \mathrm{O}_{2}(\mathrm{M}+\mathrm{H})^{+}$calc: 343.1195 obs: 343.1191 .

3 g $\left(\mathrm{R}=m\right.$ pyridyl): m.p. $226-227^{\circ} \mathrm{C} ;{ }^{1} \mathrm{H}$ NMR: ppm 8.92 (s, $1 \mathrm{H}), 8.76(\mathrm{~d}, J=3.2 \mathrm{~Hz}, 1 \mathrm{H}), 8.61(\mathrm{dd}, J=8,2.4 \mathrm{~Hz}, 1 \mathrm{H}), 8.50$ $(\mathrm{dd}, J=4.8,1.8 \mathrm{~Hz}, 1 \mathrm{H}), 8.38(\mathrm{dd}, J=7.7,1.8 \mathrm{~Hz}, 1 \mathrm{H}), 8.30$ $8.29(\mathrm{~m}, 1 \mathrm{H}), 7.46-7.44(\mathrm{~m}, 1 \mathrm{H}), 7.31(\mathrm{dd}, J=7.7,4.8 \mathrm{~Hz}, 1 \mathrm{H})$, 7.21-7.19 (m, 2H), 7.08-7.06 (m, 1H). ${ }^{13} \mathrm{C}$ NMR: ppm 172.6, $167.2,161.5,161.1,157.3,150.4,146.9,146.4,141.5,128.5,126.7$, 125.3, 125.2, 123.9, 123.0, 122.3, 121.2, 119.8; HRMS (ESI+), $m / z$ $\mathrm{C}_{18} \mathrm{H}_{12} \mathrm{~N}_{5} \mathrm{O}(\mathrm{M}+\mathrm{H})^{+}$calc: 314.1042 obs: 314.1042 .

3h $\left(\mathrm{R}=o \mathrm{FC}_{6} \mathrm{H}_{5}\right)$ : m.p. $247-249^{\circ} \mathrm{C} ;{ }^{1} \mathrm{H}$ NMR: ppm $8.59(\mathrm{~d}$, $J=7.2 \mathrm{~Hz}, 1 \mathrm{H}), 8.49-8.48(\mathrm{~m}, 1 \mathrm{H}), 7.86(\mathrm{t}, J=7.2 \mathrm{~Hz}, 1 \mathrm{H})$, 7.64-7.65 (m, 1H), $7.55(\mathrm{~d}, J=5.1 \mathrm{~Hz}, 1 \mathrm{H}), 7.41-7.35(\mathrm{~m}, 3 \mathrm{H})$, 7.13-7.03 (m, 2H), $6.86(\mathrm{~d}, J=8 \mathrm{~Hz}, 1 \mathrm{H}) ;{ }^{13} \mathrm{C}$ NMR: ppm 167.7, 156.7, 150.0, 143.7, 139.5, 137.6, 132.9, 131.8, 130.1, 126.4, 126.0, 125.1, 123.9, 123.4, 122.8, 116.6, 116.3, 115.7, 115.1; HRMS (ESI+), $m / z \mathrm{C}_{19} \mathrm{H}_{12} \mathrm{FN}_{4} \mathrm{O}(\mathrm{M}+\mathrm{H})^{+}$calc: 331.0995 obs: 331.0992 .

$31\left(\mathrm{R}=m-\mathrm{ClC}_{6} \mathrm{H}_{5}\right)$ : m.p. $236-237^{\circ} \mathrm{C}{ }^{1} \mathrm{H}$ NMR: ppm 8.59 $(\mathrm{dd}, J=7.7,1.8 \mathrm{~Hz}, 1 \mathrm{H}), 8.49-8.48(\mathrm{~m}, 1 \mathrm{H}), 7.73-7.75(\mathrm{~m}$, $1 \mathrm{H})$, 7.68-7.66 (m, $1 \mathrm{H}), 7.49-7.46(\mathrm{~m}, 3 \mathrm{H}), 7.37-7.36(\mathrm{~m}, 2 \mathrm{H})$, 7.13-7.09 (m, 1H), 6.89 (dd, $J=7.8,1.5 \mathrm{~Hz}, 1 \mathrm{H}) ;{ }^{13} \mathrm{C}$ NMR: ppm 151.2, 148.5, 142.7, 142.2, 132.9, 131.9, 130.9, 130.6, 130.4, $130.3,129.3,128.5,127.3,126.5,125.7,124.9,124.3,122.9,118.9$; HRMS (ESI+), $m / z \mathrm{C}_{19} \mathrm{H}_{12} \mathrm{ClN}_{4} \mathrm{O}(\mathrm{M}+\mathrm{H})^{+}$calc: 347.0700 obs: 347.0700 .

10: yellow oil. ${ }^{1} \mathrm{H}$ NMR: ppm 8.57 (dd, $J=7.7,1.8 \mathrm{~Hz}$, $1 \mathrm{H}), 8.48(\mathrm{dd}, J=4.8,1.8 \mathrm{~Hz}, 1 \mathrm{H}), 7.79-7.77(\mathrm{~m}, 1 \mathrm{H}), 7.63(\mathrm{~d}$, $J=8 \mathrm{~Hz}, 1 \mathrm{H}), 7.04-7.02(\mathrm{~m}, 1 \mathrm{H}), 6.87-6.85(\mathrm{~m}, 1 \mathrm{H}), 3.87(\mathrm{~s}$, 2H), 2.25 (s, 6H); ${ }^{13} \mathrm{C}$ NMR: ppm 159.9, 150.9, 149.2, 146.1, 135.6, 132.1, 131.9, 131.6, 126.6, 124.6, 123.9, 123.7, 115.3, 62.1, 46.4 HRMS (ESI+), $m / z \mathrm{C}_{16} \mathrm{H}_{15} \mathrm{ClN}_{5} \mathrm{O}(\mathrm{M}+\mathrm{H})^{+}$calc: 328.0965 obs: 328.0958 .

12: m.p. $217-219^{\circ} \mathrm{C} ;{ }^{1} \mathrm{H}$ NMR: ppm $8.77-8.75(\mathrm{~m}, 1 \mathrm{H})$, $8.48-8.43(\mathrm{~m}, 2 \mathrm{H}), 7.54(\mathrm{~d}, J=8 \mathrm{~Hz}, 1 \mathrm{H}), 7.42-7.38(\mathrm{~m}, 2 \mathrm{H})$, $3.72(\mathrm{~s}, 2 \mathrm{H}), 2.83-2.80(\mathrm{~m}, 4 \mathrm{H}), 2.57-2.55(\mathrm{~m}, 4 \mathrm{H}), 2.34$ (s, 3H) ${ }^{13} \mathrm{C}$ NMR: ppm 161.6, 151.6, 151.1, 150.1, 149.0, 139.9, 131.9, 129.9, 128.3, 126.1, 124.9, 123.0, 115.3, 55.1, 52.5, 51.7, 46.1; HRMS (ESI+), $m / z \mathrm{C}_{19} \mathrm{H}_{20} \mathrm{ClN}_{6} \mathrm{O}(\mathrm{M}+\mathrm{H})^{+}$calc: 383.1387 obs: 383.1391 .

13: m.p 191-193 ${ }^{\circ} \mathrm{C} ;{ }^{1} \mathrm{H}-\mathrm{NMR}$ : $\delta 8.57-8.54(\mathrm{~m}, 2 \mathrm{H}), 7.92$ $(\mathrm{dd}, J=5.0,0.8 \mathrm{~Hz}, 1 \mathrm{H}), 7.69(\mathrm{~d}, J=8.8 \mathrm{~Hz}, 1 \mathrm{H}), 7.63-7.58$ (m, 2H), $7.37(\mathrm{dd}, J=3.5,0.7 \mathrm{~Hz}, 1 \mathrm{H}), 7.29(\mathrm{~d}, J=2.4 \mathrm{~Hz}, 1 \mathrm{H})$, $7.24(\mathrm{dd}, J=4.9,3.8 \mathrm{~Hz}, 1 \mathrm{H}) ;{ }^{13} \mathrm{C}-\mathrm{NMR}: \delta 160.3,151.3,149.4$, $148.7,139.5,130.4,130.4,130.0,129.9,128.2,127.7,126.4,125.6$, $124.8,123.6,123.6,114.0$. HRMS (ESI+), $m / z \mathrm{C}_{17} \mathrm{H}_{9} \mathrm{ClN}_{4} \mathrm{OS}$ $(\mathrm{M}+\mathrm{H})^{+}$calc: 353.0263 obs: 353.0257 . 
14: m.p. $201-203^{\circ} \mathrm{C} ;{ }^{1} \mathrm{H}-\mathrm{NMR}: \delta 8.58-8.55$ (m, 2H), 7.98 $(\mathrm{d}, J=1.1 \mathrm{~Hz}, 1 \mathrm{H}), 7.67(\mathrm{~d}, J=8.8 \mathrm{~Hz}, 1 \mathrm{H}), 7.64-7.59(\mathrm{~m}, 2 \mathrm{H})$, 7.19 (dd, $J=3.3,0.5 \mathrm{~Hz}, 1 \mathrm{H}), 7.17(\mathrm{~d}, J=2.5 \mathrm{~Hz}, 1 \mathrm{H}), 6.81(\mathrm{dd}, J$ $=3.5,1.8 \mathrm{~Hz}, 1 \mathrm{H}) .{ }^{13} \mathrm{C}-\mathrm{NMR}: \delta 160.3,151.3,149.4,148.3,145.8$, $140.0,139.6,130.3,130.1,127.7,125.1,124.6,123.6,119.8,114.2$, 113.9, 112.3. HRMS (ESI+), $m / z \mathrm{C}_{17} \mathrm{H}_{9} \mathrm{ClN}_{4} \mathrm{O}_{2} ;(\mathrm{M}+\mathrm{H})^{+}$calc: 337.0492 obs: 337.0486 .

15: m.p 221-223 ${ }^{\circ} \mathrm{C} ;{ }^{1} \mathrm{H}-\mathrm{NMR}: \delta 8.58(\mathrm{dd}, J=7.6,1.9 \mathrm{~Hz}$, $1 \mathrm{H}), 8.46(\mathrm{dd}, J=4.8,1.9 \mathrm{~Hz}, 1 \mathrm{H}), 7.57(\mathrm{~d}, J=8.8 \mathrm{~Hz}, 1 \mathrm{H})$, $7.43-7.38(\mathrm{~m}, 3 \mathrm{H}), 7.33$ (dd, $J=8.7,2.4 \mathrm{~Hz}, 1 \mathrm{H}), 6.98(\mathrm{~d}, J=$ $2.4 \mathrm{~Hz}, 1 \mathrm{H}), 6.73(\mathrm{~d}, J=8.5 \mathrm{~Hz}, 2 \mathrm{H}), 4.07$ (s, 2H). ${ }^{13} \mathrm{C}-\mathrm{NMR}$ : $\delta 161.0,154.6,150.7,149.9,149.0,148.7,139.6,131.5,130.4,129.8$, $128.8,125.6,125.0,123.0,115.2,115.1,115.0$. HRMS (ESI+), $m / z$ $\mathrm{C}_{19} \mathrm{H}_{12} \mathrm{ClN}_{5} \mathrm{O}(\mathrm{M}+\mathrm{H})^{+}$calc: 362.0809 obs: 362.0804 .

16: m.p. $177-179^{\circ} \mathrm{C} ;{ }^{1} \mathrm{H}$ NMR: ppm 8.59 (dd, $J=7.3,1.5 \mathrm{~Hz}$, $1 \mathrm{H}), 8.49$ (dd, $J=4.81,1.8 \mathrm{~Hz}, 1 \mathrm{H}), 7.60-7.56(\mathrm{~m}, 3 \mathrm{H}), 7.45$ (d, $J=8 \mathrm{~Hz}, 2 \mathrm{H}), 7.43-7.40(\mathrm{~m}, 1 \mathrm{H}), 7.34(\mathrm{dd}, J=8.8,2.9 \mathrm{~Hz}, 1 \mathrm{H})$, $6.78(\mathrm{~d}, J=2.2 \mathrm{~Hz}, 1 \mathrm{H}), 3.60(\mathrm{~s}, 2 \mathrm{H}), 2.74-2.50(\mathrm{~m}, 8 \mathrm{H}), 2.46$ (s, 3H); ${ }^{13}$ C NMR: ppm 163.6, 153.5, 153.2, 145.2, 138.8, 136.3, 133.5, 133.2, 131.4, 131.1, 129.6, 128.9, 125.8, 125.3, 123.4, 122.9, 118.9, 62.3, 59.8, 54.7, 46.8; HRMS (ESI+), $m / z \mathrm{C}_{25} \mathrm{H}_{24} \mathrm{ClN}_{6} \mathrm{O}$ $(\mathrm{M}+\mathrm{H})^{+}$calc: 459.1700 obs: 459.1701 .

17: m.p. $241-243^{\circ} \mathrm{C} ;{ }^{1} \mathrm{H}$ NMR: ppm $8.57-8.62(\mathrm{~m}, 1 \mathrm{H})$, $8.45(\mathrm{~d}, J=1.5 \mathrm{~Hz}, 1 \mathrm{H}), 8.38-8.37(\mathrm{~m}, 1 \mathrm{H}), 7.97(\mathrm{~d}, J=8.1 \mathrm{~Hz}$, $1 \mathrm{H})$, 7.73-7.72 (m, $1 \mathrm{H}), 7.65-7.64(\mathrm{~m}, 1 \mathrm{H}), 7.59-7.58(\mathrm{~m}, 1 \mathrm{H})$, 7.49-7.48 (m, $1 \mathrm{H}), 7.17-7.16(\mathrm{~m}, 1 \mathrm{H}), 7.01(\mathrm{dd}, J=8,1.5 \mathrm{~Hz}$, $1 \mathrm{H}), 6.86(\mathrm{t}, J=7.2 \mathrm{~Hz}, 1 \mathrm{H}) ;{ }^{13} \mathrm{C}$ NMR: ppm 157.9, 153.4, $152.6,151.0,150.6,148.2,140.3,135.8,133.4,133.2,132.4,132.0$, 131.4, 128.7, 127.3, 126.7, 125.7, 124.5, 114.8; HRMS (ESI+), $m / z$ $\mathrm{C}_{19} \mathrm{H}_{12} \mathrm{~N}_{5} \mathrm{O}_{3}(\mathrm{M}+\mathrm{H})^{+}$calc: 358.0940 obs: 358.0949 .

18: m.p. 251-252 ${ }^{\circ}$; ${ }^{1} \mathrm{H}$ NMR: ppm $8.57(\mathrm{dd}, J=7.2,1.8 \mathrm{~Hz}$, $1 \mathrm{H}), 8.37$ (dd, $J=8.2,2 \mathrm{~Hz}, 1 \mathrm{H}), 7.87-7.86$ (m, $1 \mathrm{H}), 7.66-7.63$ $(\mathrm{m}, 1 \mathrm{H}), 7.52-7.49(\mathrm{~m}, 1 \mathrm{H}), 7.46-7.44(\mathrm{~m}, 2 \mathrm{H}), 7.32-7.29(\mathrm{~m}$, $1 \mathrm{H}), 7.21-7.18(\mathrm{~m}, 2 \mathrm{H}), 6.90-6.88(\mathrm{~m}, 1 \mathrm{H}) ;{ }^{13} \mathrm{C}$ NMR: ppm $167.2,165.2,163.3,160.9,153.0,148.6,143.1,138.0,131.5,131.0$, 129.6, 127.2. 127.1, 126.3, 125.4, 124.3, 122.5, 121.0, 120.8; HRMS $(\mathrm{ESI}+), m / z \quad \mathrm{C}_{19} \mathrm{H}_{12} \mathrm{FN}_{4} \mathrm{O}(\mathrm{M}+\mathrm{H})^{+}$calc: 331.0995 obs: 331.0992.

\section{Conflicts of Interest}

The authors report no conflicts of interest.

\section{Acknowledgments}

Graham B. Jones acknowledges financial support from the National Institutes of Health (UL1TR001064).

\section{References}

[1] H. Schütz, Benzodiazepines II, Springer Berlin Heidelberg, Berlin, Heidelberg, 1989.

[2] L. E. Hollister, B. Müller-Oerlinghausen, K. Rickels, and R. I. J. Shader, "Clinical uses of benzodiazepines," Journal of Clinical Psychopharmacology, vol. 13, pp. 1S-169S, 1993.

[3] J.-F. Liégeois, M. Deville, S. Dilly et al., "New pyridobenzoxazepine derivatives derived from 5-(4-methylpiperazin-1-yl) -8chloro-pyrido[2,3-b][1,5]benzoxazepine (JL13): Chemical synthesis and pharmacological evaluation," Journal of Medicinal Chemistry, vol. 55, no. 4, pp. 1572-1582, 2012.
[4] B. A. Ellenbroek and J.-F. Liégeois, "JL 13, an atypical antipsychotic: A preclinical review," CNS Drug Reviews, vol. 9, no. 1, pp. 41-56, 2003.

[5] J. F. Liegeois, F. A. Rogister, J. Bruhwyler et al., "Pyridobenzoxazepine and Pyridobenzothiazepine Derivatives as Potential Central Nervous System Agents: Synthesis and Neurochemical Study," Journal of Medicinal Chemistry, vol. 37, no. 4, pp. 519525, 1994.

[6] M. Shahid, G. B. Walker, S. H. Zorn, and E. H. F. Wong, "Asenapine: A novel psychopharmacologic agent with a unique human receptor signature," Journal of Psychopharmacology, vol. 23, no. 1, pp. 65-73, 2009.

[7] J.-F. Liégeois, A. Mouithys-Mickalad, J. Bruhwyler et al., "JL 13, a potential successor to clozapine, is less sensitive to oxidative phenomena," Biochemical and Biophysical Research Communications, vol. 238, no. 1, pp. 252-255, 1997.

[8] B. A. Ellenbroek, J. F. Liégeois, J. Bruhwyler, and A. R. J. Cools, "Effects of JL13, a pyridobenzoxazepine with potential atypical antipsychotic activity, in animal models for schizophrenia," Journal of Pharmacology and Experimental Therapeutics, vol. 298, pp. 386-391, 2001.

[9] D. E. Casey, J. Bruhwyler, J. Delarge, J. Géczy, and J.-F. Liégeois, "The behavioral effects of acute and chronic JL 13, a putative antipsychotic, in Cebus non-human primates," Psychopharmacology, vol. 157, no. 3, pp. 228-235, 2001.

[10] S. Sadler, A. R. Moeller, and G. B. Jones, "Microwave and continuous flow technologies in drug discovery," Expert Opinion on Drug Discovery, vol. 7, no. 12, pp. 1107-1128, 2012.

[11] J. F. da Costa, X. García-Mera, O. Caamano, J. M. Brea, M. I. Loza, and J. Med, "Synthesis by microwave-assisted 1,3-dipolar cycloaddition of 1,2,3-triazole 1'-homo-3'-isoazanucleosides and evaluation of their anticancer activity," European Journal of Medicinal Chemistry, vol. 98, pp. 212-220, 2015.

[12] I. A. Al-Masoudi, Y. A. Al-Soud, N. J. Al-Salihi, and N. A. AlMasoudi, "1,2,4-Triazoles: Synthetic approaches and pharmacological importance. (Review)," Chemistry of Heterocyclic Compounds, vol. 42, no. 11, pp. 1377-1403, 2006.

[13] G. Pellizzari, "Triazolo e suoi derivati," Gazzetta Chimica Italiana, vol. 41, p. 20, 1911.

[14] S. Sadler, M. Sebeika, N. Kern et al., "A facile route to triazolopyrimidines using a [3+2] cycloaddition and continuousflow chemistry," Journal of Flow Chemistry, vol. 4, no. 3, pp. 140147,2014

[15] K. Kanagarajadurai, M. Malini, A. Bhattacharya, M. M. Panicker, and R. Sowdhamini, "Molecular modeling and docking studies of human 5-hydroxytryptamine 2A (5-HT2A) receptor for the identification of hotspots for ligand binding," Molecular BioSystems, vol. 5, no. 12, pp. 1877-1888, 2009.

[16] M. N. Jadhav, G. R. Kokil, S. S. Harak, and S. B. Wagh, "Direct and indirect drug design approaches for the development of novel tricyclic antipsychotics: Potential 5-HT2A antagonist," Journal of Chemistry, vol. 2013, Article ID 930354, pp. 1-8, 2013.

[17] H. Y. Meltzer, S. Matsubara, J. C. Lee, and J. Pharmacol, "Classification of typical and atypical antipsychotic drugs on the basis of dopamine D-1, D-2 and serotonin2 pKi values," Journal of Pharmacology and Experimental Therapeutics, vol. 251, no. 1, pp. 238-246, 1989.

[18] R. G. Booth, L. Fang, Y. Huang, A. Wilczynski, and S. Sivendran, "For binding protocols see:" European Journal of Pharmacology, vol. 1, no. 3, pp. 1-9, 2009. 
[19] P. LaBeaume, M. Placzek, M. Daniels et al., "Microwaveaccelerated fluorodenitrations and nitrodehalogenations: expeditious routes to labeled PET ligands and fluoropharmaceuticals," Tetrahedron Letters, vol. 51, no. 14, pp. 1906-1909, 2010.

[20] K. S. Elvira, X. Casadevali i Solvas, R. C. Wootton, and A. J. deMello, "For additional applications of flow chemistry see:" Nature Chemistry, vol. 5, pp. 905-915, 2013.

[21] S. V. Ley, D. E. Fitzpatrick, R. M. Myers, C. Battilocchio, and R. J. Ingham, "Machine-Assisted Organic Synthesis," Angewandte Chemie International Edition, vol. 54, no. 35, pp. 10122-10136, 2015. 

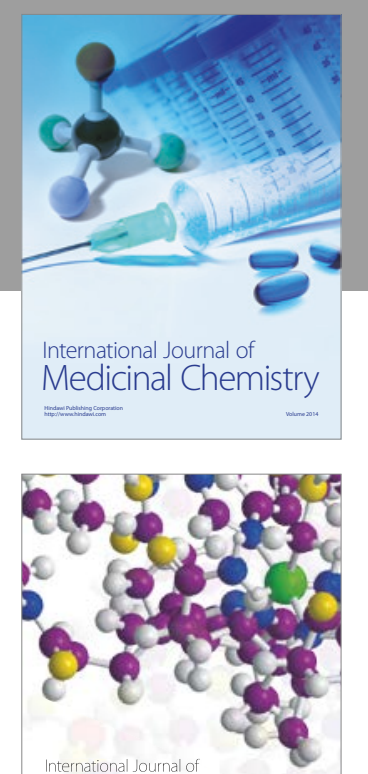

Carbohydrate Chemistry

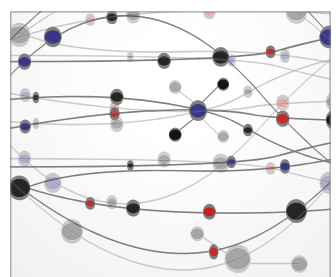

The Scientific World Journal
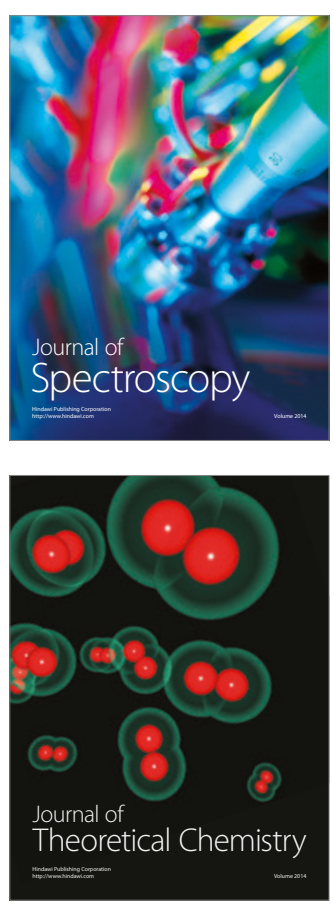
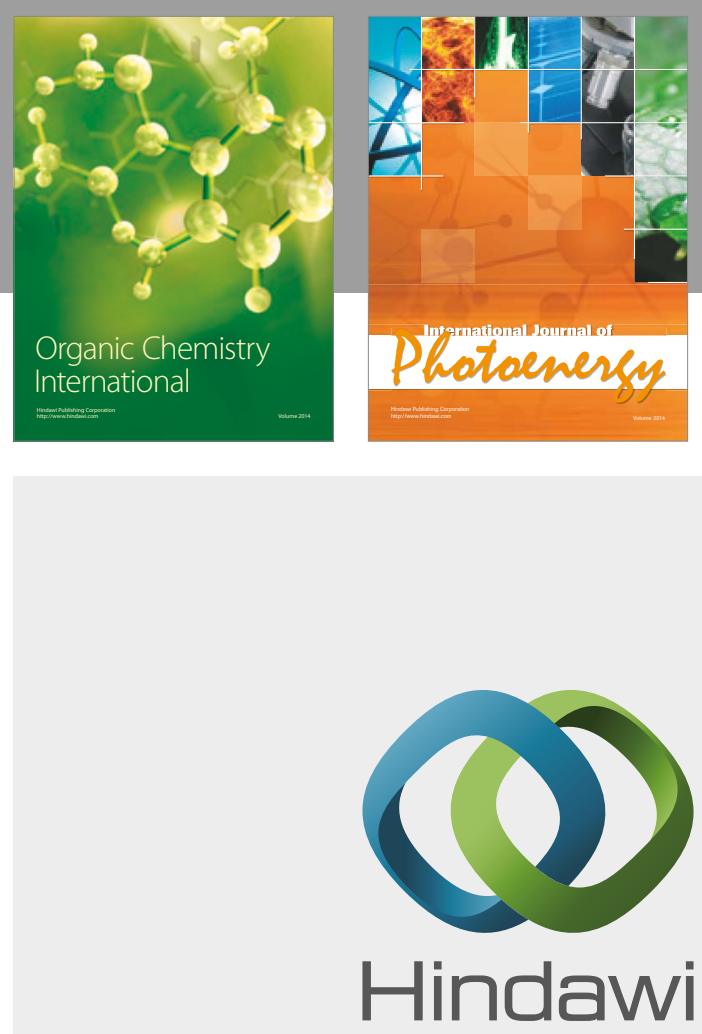

Submit your manuscripts at

https://www.hindawi.com

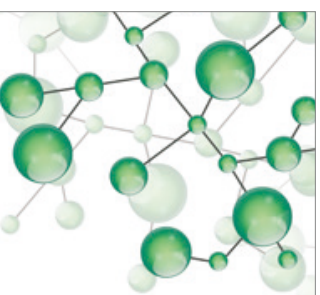

International Journal of

Inorganic Chemistry

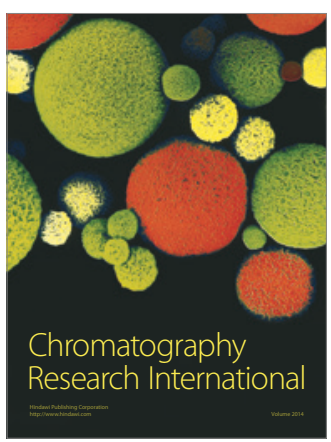

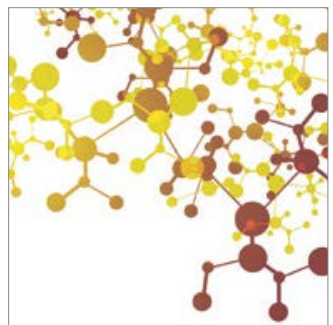

Applied Chemistry
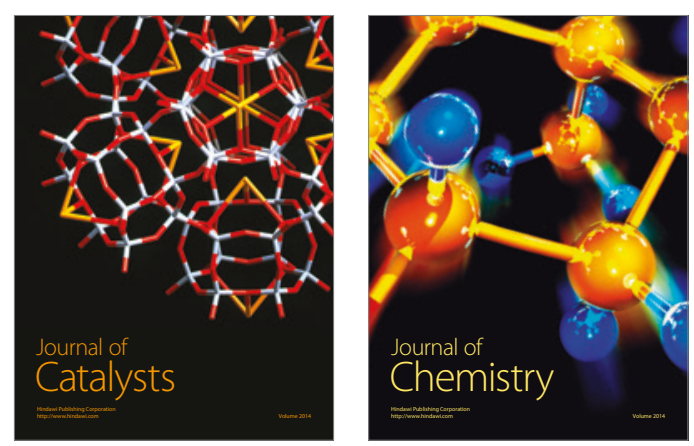
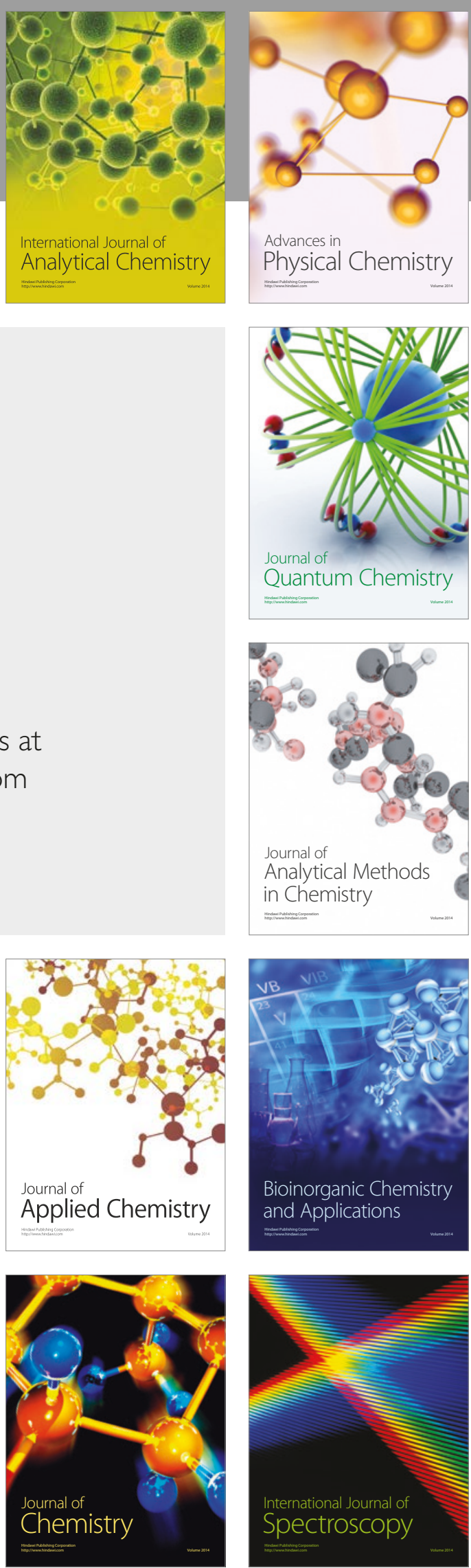